\title{
Cinétique d'infestation par les tiques des bovins de la région subhumide de Constantine en Algérie
}

\author{
Mohamed Chérif Benchikh Elfegoun ${ }^{1 *}$ Karima Kohil $^{1}$ \\ Mohamed Gharbi ${ }^{2}$ Larbi Afoutni ${ }^{1}$ \\ Mohamed Lamine Benachour ${ }^{1}$
}

\section{Mots-clés}

Bovin, Metastigmata, Ixodidae, Rhipicephalus bursa, Hyalomma scupense, piroplasmose, Algérie

Submitted: 18 November 2018

Accepted: 8 April 2019

Published: 20 May 2019

DOI: $10.19182 /$ remvt.31726

\section{Résumé}

Une étude a été menée de mai 2008 à avril 2009 pour déterminer une fois par mois dans deux élevages de la région de Constantine (étage subhumide de I'Algérie), situés respectivement à 500 et 1000 mètres d'altitude, l'évolution de l'infestation des bovins par les tiques. Sur quarante bovins suivis (20 par élevage), 2031 tiques ont été récoltées sur les différentes régions anatomiques (périnée, mamelle, scrotum, oreilles, entre autres). Sept espèces de tiques réparties en quatre genres ont été identifiées : Rhipicephalus bursa (48,5\%), tiques du groupe R. sanguineus (26,6\%), Hyalomma marginatum (11\%), Haemaphysalis punctata (5,7\%), Hy. lusitanicum (3,9\%), Hy. scupense (2,7\%) et Ixodes ricinus $(1,5 \%)$. La prédominance de $R$. bursa, d'une part, et l'absence de $H$. scupense à $1000 \mathrm{~m}$ d'altitude, d'autre part, ont été notées. Par ailleurs, l'influence de I'altitude et de l'âge a été étudiée, et la localisation des tiques sur les animaux a été enregistrée. L'étude de la dynamique saisonnière d'infestation a montré que la lutte antivectorielle, en cas de fortes infestations, devrait être principalement appliquée durant la période chaude (de mai à août), correspondant au pic d'abondance de $R$. bursa et de $H$. scupense, principales espèces responsables de la transmission des piroplasmoses chez les bovins, dont les babésioses et la theilériose tropicale.

- Comment citer cet article : Benchikh Elfegoun M.C., Kohil K., Gharbi M., Afoutni L., Benachour M.L., 2019. Kinetics of tick infestation in cattle in subhumid Constantine region in Algeria. Rev. Elev. Med. Vet. Pays Trop., 72 (1): 41-45, doi: 10.19182/remvt.31726

\section{INTRODUCTION}

Les tiques sont des arthropodes hématophages parasitant tous les vertébrés à travers le monde. Outre la spoliation de sang, elles sont à l'origine de lésions cutanées, inoculant des dizaines de molécules dont certaines sont immunodépressives, anti-inflammatoires, anesthésiantes, hémolysantes ou anticoagulantes. Certaines espèces produisent des toxines paralysantes ou causent des eczémas importants (dyshidrose à tiques). De plus, elles interviennent dans la transmission

\footnotetext{
1. Laboratoire de parasitologie, Institut des sciences vétérinaires, Université 1, Constantine, Algérie.

2. Laboratoire de parasitologie, Ecole nationale de médecine vétérinaire, Université de la Manouba, Sidi Thabet, Tunisie.

* Auteur pour la correspondance

Email : blmc25@yahoo.fr
}

de maladies potentiellement graves dont les piroplasmoses qui constituent une contrainte pour le développement et l'amélioration génétique du cheptel bovin dans le monde. En Algérie, les piroplasmoses, au sens large, présentes dans les élevages bovins sont principalement les babésioses et la theilériose tropicale dues respectivement à Babesia bovis, B. bigemina et Theileria annulata, dont les vecteurs principaux sont Rhipicephalus bursa, R. annulatus et Hyalomma scupense (Sergent et al., 1945). Plus de la moitié des cas cliniques de piroplasmoses diagnostiqués relèvent de la theilériose tropicale (Sergent et al., 1945 ; Ziam et Benaouf, 2004 ; Benchikh Elfegoun et al., 2017).

La détermination de la période d'activité des tiques vectrices des piroplasmoses bovines dans une région donnée permet d'établir un programme de lutte antivectorielle adapté. L'objet de la présente étude a été d'inventorier les espèces de tiques parasites des bovins dans la région de Constantine (étage subhumide du nord-est de l'Algérie) et d'étudier l'influence de l'altitude sur l'intensité d'infestation des animaux par les tiques. 


\section{MATERIEL ET METHODES}

\section{Région de l'étude}

La wilaya de Constantine est située entre $36,1^{\circ}$ et $36,6^{\circ} \mathrm{N}$, et $6,4^{\circ}$ et $7,1^{\circ} \mathrm{E}$, à l'est de l'Algérie et à 80 kilomètres du littoral de la mer Méditerranée (figure 1). Elle est limitée à l'ouest par la wilaya de Mila, au nord par celle de Skikda, à l'est par celle de Guelma, et au sud par celle d'Oum El-Bouaghi. C'est une région charnière entre le Tell (région côtière) et les Hauts Plateaux, avec une prédominance de reliefs de collines. Elle est traversée par deux oueds, le Rhummel au nord et le Boumerzoug au sud. La wilaya s'étend sur 2229 kilomètres carrés.

Sur le plan climatique, la wilaya de Constantine bénéficie d'un climat méditerranéen subhumide et d'une pluviométrie moyenne annuelle évaluée à $525 \mathrm{~mm}$. Il y règne deux étages bioclimatiques différenciés : une zone Sud recevant en moyenne $400 \mathrm{~mm}$ de précipitations, qui s'apparente aussi bien par le relief que par le climat à la région des Hauts Plateaux ; et une zone Nord bénéficiant d'une pluviométrie moyenne de $600 \mathrm{~mm}$, marquée par un relief plus ou moins chahuté et rattachée à la zone tellienne qui sépare les hautes plaines du littoral. La température maximale est de $39,7^{\circ} \mathrm{C}$ avec des pics dépassant $40^{\circ} \mathrm{C}$ et la température minimale est de $-5,4^{\circ} \mathrm{C}$. La saison froide et pluvieuse s'étend de la mi-octobre à la mi-avril, suivie d'une période chaude et sèche (Station météorologique d'Aïn El Bey, 2017).

\section{Animaux}

L'étude a été réalisée dans deux élevages de la wilaya situés l'un à 500 mètres et l'autre à 1000 mètres d'altitude. Dans chacun d'eux, 20 animaux ont été sélectionnés par tirage au sort de façon à ce qu'au total il y ait 20 bovins de race locale (Brune de l'Atlas) et 20 bovins croisés (avec la Frisonne Pie noire), avec une répartition identique dans les deux fermes (10 âgés de moins d'un an et 10 plus âgés pour chaque race) (tableau I).

\section{난 Collecte des tiques}

Les tiques ont été récoltées sur les bovins une fois par mois de mai 2008 à avril 2009. Au cours de l'étude, aucun traitement acaricide n'a été appliqué sur les animaux. Toutes les tiques prélevées ont été conservées dans de l'alcool à $70^{\circ}$. Au laboratoire, l'identification des tiques a été faite à la loupe binoculaire selon les clés publiées par Bouattour (2002), et Walker et al. (2003).

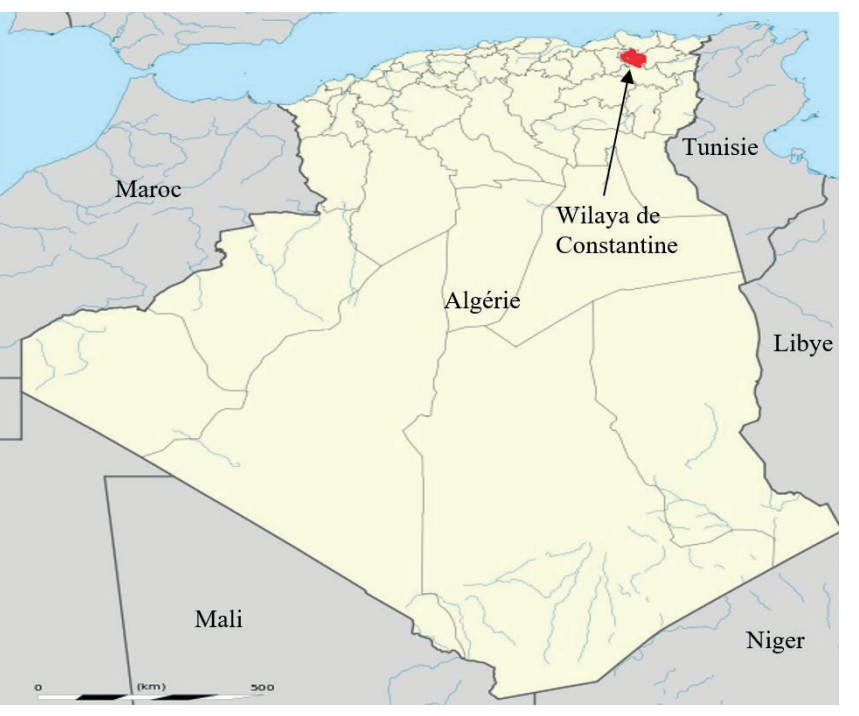

Figure 1 : situation géographique de la région de l'étude.

\section{Analyses statistiques}

Les comparaisons portant sur le nombre moyen de tiques récoltées ont été effectuées au moyen du test U de Mann et Whitney, lorsqu'il s'agissait de comparer des séries de valeurs recueillies dans deux classes (bovins de moins d'un an contre bovins de plus d'un an), et au moyen du test $\mathrm{H}$ de Kruskal-Wallis lorsqu'il s'agissait de comparer des séries de valeurs sur plus de deux classes (nombre de tiques récoltées chaque mois). Le risque alpha retenu pour rejeter l'hypothèse nulle était de 0,05 .

\section{RESULTATS}

\section{Espèces de tiques récoltées}

L'étude a permis de récolter 2031 tiques adultes et 970 tiques immatures (951 nymphes et 19 larves), toutes espèces confondues. Parmi les tiques adultes, 536 ont été récoltées sur les animaux de la ferme située à $500 \mathrm{~m}$ d'altitude et 1495 sur ceux de la ferme installée à $1000 \mathrm{~m}$.

Sept espèces de tiques, appartenant à quatre genres, ont été identifiées. Il s'agissait de Rhipicephalus bursa (qui représentait 48,5\% des tiques récoltées), des tiques du groupe $R$. sanguineus (26,6\%), de Hyalomma marginatum (11,0\%), de Haemaphysalis punctata (5,7\%), de Hyalomma lusitanicum (3,9\%), de Hyalomma scupense $(2,7 \%)$ et d'Ixodes ricinus (1,5\%) (tableau II). L'identification des formes immatures n'a pas été possible car la majorité des spécimens récoltés étaient abîmés.

\section{Tableau I}

Distribution des animaux suivis selon la race, l'âge et I'altitude de la ferme dans le Nord-Est algérien

\begin{tabular}{llcc} 
& & \multicolumn{2}{c}{ Altitude (m) } \\
Type de bovins & Age & $\mathbf{5 0 0}$ & $\mathbf{1 0 0 0}$ \\
\hline Locaux & $<1$ an & 5 & 5 \\
& $\geq 1$ an & 5 & 5 \\
\hline Croisés & $<1$ an & 5 & 5 \\
& $\geq 1$ an & 5 & 5
\end{tabular}

\section{Tableau II}

Fréquence des espèces de tiques selon I'altitude dans deux fermes du Nord-Est algérien

\begin{tabular}{lccc} 
Espèces de tiques & $\begin{array}{c}\text { Elevage } \\
\text { à } \mathbf{5 0 0} \mathbf{~ m} \\
\text { d'altitude }\end{array}$ & $\begin{array}{c}\text { Elevage } \\
\text { à } \mathbf{1 0 0 0} \mathbf{~ m} \\
\text { d'altitude }\end{array}$ & Total \\
& $\mathbf{N b .} \mathbf{( \% )}$ & $\mathbf{N b .}(\mathbf{\%})$ & $\mathbf{N b .}(\mathbf{\%})$ \\
\hline $\begin{array}{l}\text { Rhipicephalus bursa } \\
\text { Rhipicephalus du groupe }\end{array}$ & $122(22,8)$ & $863(57,7)$ & $985(48,5)$ \\
sanguineus & $184,3)$ & $356(23,8)$ & $540(26,6)$ \\
Hyalomma scupense & $56(10,4)$ & - & $56(2,7)$ \\
Hyalomma lusitanicum & $53(9,9)$ & $27(1,8)$ & $80(3,9)$ \\
Hyalomma marginatum & $87(16,2)$ & $137(9,2)$ & $224(11)$ \\
Haemaphysalis punctata & $23(4,3)$ & $92(6,2)$ & $115(5,7)$ \\
Ixodes ricinus & $11(2,1)$ & $20(1,3)$ & $31(1,5)$ \\
Total & $536(26,4)$ & $1495(73,6)$ & $2031(100)$
\end{tabular}




\section{Influence de l'altitude}

L'influence de l'altitude a été observée : 73,6\% des tiques ont été récoltées sur les bovins élevés à $1000 \mathrm{~m}$ d'altitude et $26,4 \%$ sur ceux vivant à $500 \mathrm{~m}$. Les espèces prédominantes étaient d'autre part différentes selon l'altitude. $R$. bursa représentait la majorité des tiques $(57,7 \%)$ récoltées à $1000 \mathrm{~m}$, mais seulement $22,8 \%$ de celles récoltées à $500 \mathrm{~m}$ où les tiques du groupe $R$. sanguineus étaient prédominantes (34,3\% contre 23,8 \% à $1000 \mathrm{~m})$. Les autres espèces étaient généralement plus fréquentes à $500 \mathrm{~m}$ qu'à $1000 \mathrm{~m}$ (H. lusitanicum : $9,9 \%$ contre $1,8 \%$; H.marginatum : $16,2 \%$ contre $9,2 \%$; I. ricinus : $2 \%$ contre $1,3 \%$ ) à l'exception de Hae. punctata dont la fréquence était légèrement plus élevée à $1000 \mathrm{~m}(6,1 \%$ contre 4,3 \%). Par ailleurs, $H$. scupense a été récoltée uniquement à basse altitude où elle représentait 10,4\% des tiques (tableau II).

\section{Influence de l'âge}

L'influence de l'âge des animaux a été également notée puisque 91,8\% des tiques ont été récoltées sur les bovins âgés et seulement $8,2 \%$ sur les jeunes bovins. L'influence de l'âge sur l'intensité d'infestation était très significative indépendamment du mois de l'année ( $p<0,0001$; test U de Mann-Whitney) (tableau III).

\section{Fréquence des espèces de tiques selon la saison ou dynamique annuelle}

Pendant toute la période chaude (d'avril à août), la charge parasitaire mensuelle par les tiques adultes, toutes espèces confondues, était largement supérieure sur les bovins élevés à $1000 \mathrm{~m}$ que sur ceux vivant en basse altitude ( $p<0,0001$; test $\mathrm{H}$ de Kruskal-Wallis). L'infestation était maximale durant cette période indépendamment de l'altitude (figure 2).

Les adultes de $R$. bursa ont été observés sur les bovins entre avril et septembre, l'infestation ayant été plus importante dans l'élevage à $1000 \mathrm{~m}$ d'altitude (figure 3). Plus de la moitié (32 sur 56 au total) des adultes de $H$. scupense ont été récoltés sur les animaux au mois de juillet (figure 4). Les formes immatures, principalement des nymphes, ont été récoltées d'octobre à décembre à basse altitude et d'octobre à

\section{Tableau III}

Influence de l'âge sur l'infestation des bovins par les tiques dans deux fermes du Nord-Est algérien

\section{Nb. moyen de tiques / animal ( \pm ET *)}

\section{Bovins $<1$ an}

(167 tiques)

Bovins $\geq 1$ an (1864 tiques)

$\begin{array}{lcc}\text { Mai } & 4,4 \pm 1,9(87) & 11,1 \pm 4,6(221) \\ \text { Juin } & 1,4 \pm 1,2(28) & 24,3 \pm 15,9(485) \\ \text { Juillet } & 0,6 \pm 0,7(11) & 15,3 \pm 5,8(305) \\ \text { Août } & 0,5 \pm 0,5(9) & 10,7 \pm 5,9(214) \\ \text { Septembre } & 0,1 \pm 0,3(2) & 2,4 \pm 2,1(48) \\ \text { Octobre } & 0,1 \pm 0,2(1) & 3,8 \pm 3,1(76) \\ \text { Novembre } & 0,1 \pm 0,2(1) & 1,1 \pm 0,9(22) \\ \text { Décembre } & 0,1 \pm 0,2(1) & 0,8 \pm 0,8(15) \\ \text { Janvier } & 0 & 0,8 \pm 0,8(15) \\ \text { Février } & 0,1 \pm 0,2(1) & 0,1 \pm 0,3(2) \\ \text { Mars } & 0,3 \pm 0,5(5) & 1,7 \pm 0,5(34) \\ \text { Avril } & 1,1 \pm 0,8(21) & 21,4(427)\end{array}$

* Ecart-type

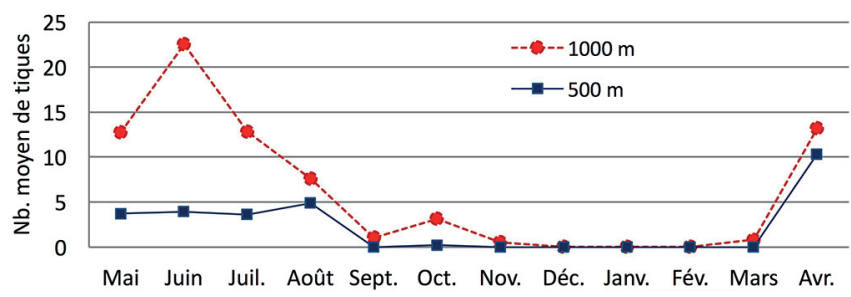

Figure 2 : évolution mensuelle de la charge parasitaire moyenne des bovins selon I'altitude de la ferme dans le Nord-Est algérien.

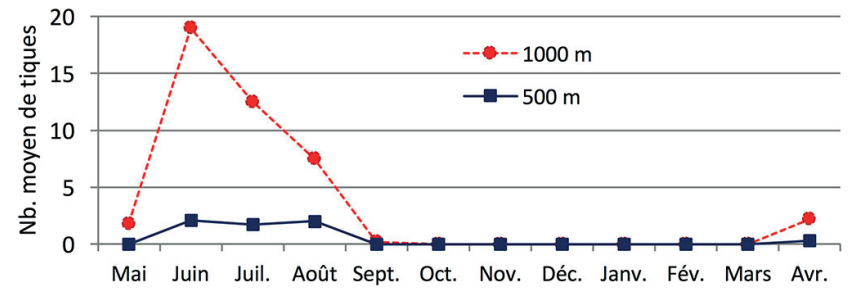

Figure 3 : évolution mensuelle du nombre moyen de Rhipicephalus bursa infestant les bovins selon l'altitude de la ferme dans le Nord-Est algérien.

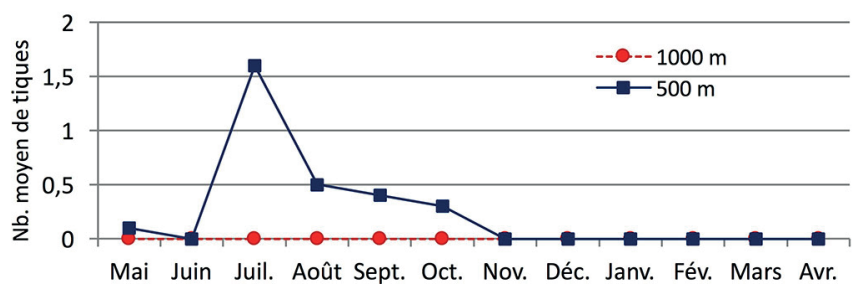

Figure 4 : évolution mensuelle du nombre moyen de Hyalomma scupense infestant les bovins selon l'altitude de la ferme dans le Nord-Est algérien.

février à haute altitude ; le nombre moyen de nymphes variait de 1,60 à 5,45 tiques/animal et de 0,8 à 19,4 tiques/animal respectivement à $500 \mathrm{~m}$ et $1000 \mathrm{~m}$. Quelques larves ont été aussi prélevées sur les bovins au cours de notre enquête.

\section{Régions du corps infestées}

Les tiques adultes ont été retrouvées sur six régions du corps avec cependant une plus forte infestation de la mamelle et du scrotum pour l'ensemble des espèces de tiques identifiées, à l'exception de celles du groupe $R$. sanguineus. Ces dernières se fixaient principalement au niveau des oreilles (tableau IV). Les autres régions du corps (fanon et cou, ventre, aisselles, flanc, pattes, onglons et paturons) n'étaient pas infestées. Les formes immatures ont été surtout observées au niveau de la mamelle.

\section{DISCUSSION}

Cette étude a montré une nette prédominance de $R$. bursa dans la région de Constantine. Cette tique, vectrice de Babesia bovis et de B. bigemina (Sergent et al., 1945 ; Gayot, 1953 ; El Hadj et al., 2002), est présente dans les régions à végétation basse, herbacée ou buissonnante. La fréquence élevée de $R$. bursa dans les deux grandes régions naturelles de l'Algérie, le littoral et les Hauts-Plateaux, a été rapportée par Sergent et al. en 1945 (plus de $44 \%$ des tiques récoltées sur les bovins étaient des R. bursa). 


\section{Tableau IV}

Fréquence des espèces de tiques selon la localisation anatomique chez les bovins dans deux fermes du Nord-Est algérien

\begin{tabular}{|lccccccc} 
Localisation anatomique & R. bursa & $\begin{array}{c}\text { R. du groupe } \\
\text { sanguineus }\end{array}$ & H. scupense & H. marginatum & H. lusitanicum & Hae. punctata & I. ricinus \\
Oreilles & 6,1 & 76,9 & 1,8 & 7,1 & 11,3 & 4,3 \\
Paupières & 0,1 & 0,4 & 0 & 0,9 & 0 & 0 & 22,6 \\
Dos & 0 & 0,7 & 0 & 0 & 0 & 1,7 & 6,5 \\
Face interne des cuisses & 6,2 & 3,9 & 21,4 & 23,7 & 15,0 & 53,0 & 29,0 \\
Mamelle et scrotum & 68,5 & 15,9 & 51,8 & 53,6 & 62,5 & 39,1 & 35,5 \\
Périnée & 18,3 & 0,7 & 25,0 & 10,3 & 11,3 & 0,9 & 6,5 \\
Base de la queue & 0,8 & 1,5 & 0 & 4,5 & 0 & 0,9
\end{tabular}

Malgré la prédominance de cette espèce à 1000 m d'altitude, les cas cliniques de babésioses bovines sont rares selon les services vétérinaires. Cette absence de piroplasmoses cliniques pourrait s'expliquer par l'installation d'une enzootie stable due au développement de l'immunité chez les bovins (Morel, 2003). Darghouth et al. (1996) ont décrit cet état de stabilité enzootique dans des élevages traditionnels tunisiens. En automne, après leur première saison d'infestation par les tiques et d'infection par les agents pathogènes qu'elles transmettent, les jeunes bovins étaient tous séropositifs à Theileria annulata. Ainsi, dans cet état d'endémie, les cas cliniques rapportés feraient suite à une mauvaise prise colostrale, une infestation par plusieurs tiques infectantes, une infection par des souches particulièrement virulentes, une immunodépression, ou toucheraient les animaux nouvellement introduits (Gharbi et al., 2014). Dans la présente étude, des infestations élevées par les tiques adultes $R$. bursa ( $>18$ tiques / bovin adulte) ont été observées à $1000 \mathrm{~m}$ d'altitude, favorisant sans doute l'installation d'une enzootie stable.

En revanche, à basse altitude, la présence de $R$. bursa et de $H$. scupense se traduit par l'apparition de nombreux cas de babésioses et de theilériose tropicale dans les élevages bovins de la région (Direction de la santé animale, wilaya de Constantine, 2008). Cette situation peut s'expliquer par l'absence de stabilité enzootique due à une infestation trop faible par les tiques adultes lors du pic d'infestation $(<2$ tiques / bovin adulte en juin et juillet).

Dans la présente étude, l'influence marquée de l'altitude (trois quarts des tiques ont été récoltées à 1000 m) a été observée. Les animaux de basse altitude avaient été traités contre les tiques au cours des années précédentes, contrairement à ceux de l'autre élevage. Cela pourrait expliquer la plus faible infestation lors de l'année d'étude. L'enquête a montré également que l'influence de l'âge pouvait résulter du fait que les jeunes bovins vivaient généralement en stabulation et étaient moins exposés aux infestations par les tiques que les sujets adultes. Ces résultats corroborent ceux obtenus dans la région humide de Taher (Benchikh Elfegoun et al., 2007).

L'étude a confirmé l'existence d'une dynamique saisonnière unimodale et d'une activité maximale de $R$. bursa en été (avec un pic d'infestation en juin) et de $H$. scupense en été et début automne (avec un pic en juillet). Le caractère estival de ces deux espèces a été rapporté par Morel (1981 ; 2003), Bouattour (1996) en Tunisie, et Sahibi et Rhalem (2007) au Maroc, où les adultes de H. scupense et $R$. bursa présentent la même activité intense pendant les mois secs et chauds.

\section{CONCLUSION}

A la lumière des résultats relatifs à la fréquence et à la dynamique saisonnière des principales espèces de tiques (H. scupense et $R$. bursa) impliquées dans la transmission des piroplasmoses bovines, les programmes de lutte antivectorielle ne devront être appliqués qu'en cas d'infestations massives des animaux, lors des années favorables à la pullulation des tiques et durant la période d'intense activité des vecteurs de piroplasmoses sensu lato, s'étalant de mai à septembre. Le but du traitement acaricide sera alors de diminuer la pression parasitaire sans empêcher l'immunité de prémunition de se développer, assurant ainsi une stabilité enzootique durable. L'intervention doit également tenir compte des mœurs particulières de $H$. scupense, tique endophile : il est nécessaire d'assurer le crépissage et le blanchiment des murs des locaux d'hébergement des animaux pour supprimer les gîtes de repos des nymphes et des jeunes adultes de cette espèce.

\section{Déclaration des contributions des auteurs}

MCBE et KK ont participé à la conception et à la planification de l'étude ; MCBE, LA et MLB ont effectué la collecte des tiques sur le terrain ; MCBE et KK ont procédé à l'identification des tiques et l'interprétation des données obtenues ; MCBE a recueilli les données et rédigé la première version du manuscrit ; MG a effectué les analyses statistiques et révisé le manuscrit.

\section{REFERENCES}

Benchikh Elfegoun M.C., Benakhla A., Bentounsi B., Bouattour A., Piarroux R., 2007. Identification et cinétique saisonnière des tiques parasites des bovins dans la région de Taher (Jijel), Algérie. Ann. Med. Vet., 151 (4) : 209-214

Benchikh Elfegoun M.C., Gharbi M., Merzekani Z., Kohil K., 2017. Bovine piroplasmosis in the provinces of Skikda and Oum El Bouaghi (Northeastern Algeria): Epidemiological study and estimation of milk yield losses. Rev. Elev. Med. Vet. Pays Trop., 70 (3): 105-110, doi: 10.19182/ remvt.31519

Bouattour A., 1996. Etude des tiques des bovins dans la région de Sidi Thabet : leur rôle dans la transmission de la theilériose. Diplôme études approf. écol. anim., Faculté des sciences de Tunis, Tunisie, 62 p.

Bouattour A., 2002. Clé dichotomique et identification des tiques (Acari : Ixodidae) parasites du bétail au Maghreb. Arch. Inst. Pasteur Tunis, 79 $(1-4): 43-50$

Darghouth M.A., Bouattour A., Ben Miled L., Kilani M., Brown C.G.D., 1996. Epidemiology of tropical theileriosis (Theileria annulata infection of cattle) in an endemic region of Tunisia: characterisation of endemicity states. Vet. Parasitol., 65 (3-4): 199-211, doi: 10.1016/S0304-4017(96)00974-0 
Direction de la santé animale, 2008. Rapport annuel. Wilaya de Constantine, Algérie, $25 \mathrm{p}$.

El Haj N., Kachani M., Bouslikhane M., Ouhelli H., Ahami A.T., Katende J., Morzaria S.P., 2002. Séroépidémiologie de la theilériose et de la babésiose au Maroc. Rev. Méd. Vét., $153:$ 809-814

Gayot G., 1953. Les maladies du cheptel en Tunisie en été. Tunisie Agric., $10: 151-162$

Gharbi M., Darghouth M.A., 2014. A review of Hyalomma scupense (Acari, Ixodidae) in the Maghreb region: from biology to control. Parasite, 21: 2, doi: 10.1051/PARASITE/2014002

Morel P.C., 1981. Maladies à tiques du bétail en Afrique. In: Précis de parasitologie vétérinaire tropicale (Ed. Troncy P.M., Itard J., Morel P.C.). Ministère de la Coopération et du Développement, Paris, France, 471-717

Morel P.C., 2003. Les tiques d'Afrique et du Bassin méditerranéen. CD-ROM, Cirad, Montpellier, France
Sahibi H., Rhalem A., 2007. Tiques et maladies transmises par les tiques chez les bovins au Maroc. Institut agronomique et vétérinaire Hassan II, Rabat, Maroc. Bull. Mens. Liaison Inf. PNTTA (151) : 1-4

Sergent E., Donatien A., Parrot L., Lestoquard F., 1945. Etudes sur les piroplasmoses bovines. Arch. Inst. Pasteur Algérie, 816 p.

Station météorologique d'Aïn El Bey, 2017. Rapport annuel. Wilaya de Constantine, Algérie, $18 \mathrm{p}$

Walker A.R., Bouattour A., Camicas J.-L., Estrada-Peña A., Horak I.G., Latif A.A., Pegram R.G., et al., 2003. Ticks of domestic animals in Africa. A guide to identification of species. University of Edinburgh, Edinburgh, UK, $227 \mathrm{p}$.

Ziam H., Benaouf H., 2004. Prevalence of blood parasites in cattle from wilayates of Annaba and El Tarf in East Algeria. Arch. Inst. Pasteur Tunis, 81 (1-4): $27-30$

\section{Summary}

Benchikh Elfegoun M.C., Kohil K., Gharbi M., Afoutni L., Benachour M.L. Kinetics of tick infestation in cattle in subhumid Constantine region in Algeria

A study was conducted from May 2008 to April 2009 to monitor monthly the tick infestation of cattle in two farms in Constantine region (subhumid area of Algeria), located at 500 and $1000 \mathrm{~m}$ of altitude, respectively. Out of forty monitored cattle (20 per farm), 2031 ticks were collected from different anatomical sites (perineum, udder, scrotum, ears, among others). Seven tick species from four genera were identified: Rhipicephalus bursa (48.5\%), ticks of $R$. sanguineus group (26.6\%), Hyalomma marginatum (11\%), Haemaphysalis punctata $(5.7 \%)$, Hy. lusitanicum (3.9\%), Hy. scupense (2.7\%), and Ixodes ricinus (1.5\%). The predominance of $R$. bursa on the one hand and the absence of Hy. scupense at $1000 \mathrm{~m}$ altitude on the other hand were noted. Furthermore, the influences of altitude and age were analyzed and tick location on the animals was recorded. The study of seasonal infestation dynamics showed that vector control, in case of heavy infestations, should be mainly applied during the warm period (May to August), corresponding to the peak of abundance of $R$. bursa and $H$. scupense, the main species responsible for the transmission of piroplasmoses in cattle, including babesioses and tropical theileriosis.

Keywords: cattle, Metastigmata, Ixodidae, Rhipicephalus bursa, Hyalomma scupense, piroplasmosis, Algeria

\section{Resumen}

Benchikh Elfegoun M.C., Kohil K., Gharbi M., Afoutni L., Benachour M.L. Cinética de la infestación por garrapatas en el ganado en la región subhúmeda de Constantino en Argelia

Se llevó a cabo un estudio de mayo de 2008 a abril de 2009, con el fin de monitorear mensualmente la infestación de garrapatas en bovinos en dos fincas de la región de Constantino (área subhúmeda de Argelia), ubicadas a 500 y 1000 metros de altitud, respectivamente. De cuarenta bovinos (20 por finca) monitoreados, se recolectaron 2031 garrapatas de diferentes regiones anatómicas (perineo, ubre, escroto, orejas, entre otras). Se identificaron siete especies de garrapatas de cuatro géneros: Rhipicephalus bursa (48,5\%), garrapatas del grupo R. sanguineus (26,6\%), Hyalomma marginatum (11\%), Haemaphysalis punctata $(5,7 \%)$, Hy. lusitanicum $(3,9 \%)$, Hy. scupense $(2,7 \%)$ e Ixodes ricinus $(1,5 \%)$. Se notó el predominio de $R$. bursa por un lado y la ausencia de Hy. scupense a $1000 \mathrm{~m}$ de altitud por el otro. Se analizaron además la influencia de la altitud y la edad y se registró la ubicación de las garrapatas en los animales. El estudio de la dinámica de la infestación estacional mostró que el control de vectores, en caso de infestaciones graves, debe aplicarse principalmente durante el período cálido (de mayo a agosto), correspondiente al pico de abundancia de $R$. bursa y $H$. scupense, la principal especie responsable para la transmisión de piroplasmosis en el ganado, incluidas las babesiosis y la teileriosis tropical.

Palabras clave: ganado bovino, Metastigmata, Ixodidae, Rhipicephalus bursa, Hyalomma scupense, piroplasmosis, Argelia 
